\title{
Stable Vicinal Step Orientations in $m$-Plane GaN
}

\author{
K.M. Kelchner ${ }^{\text {a }}$, L.Y. Kuritzky ${ }^{\mathrm{a}, 1}$, S. Nakamura ${ }^{\mathrm{a}, \mathrm{b}}$, S.P. DenBaars ${ }^{\text {a,b }}$ and J.S. Speck ${ }^{\mathrm{a}}$ \\ ${ }^{a}$ Materials Department, University of California, Santa Barbara, 93106 USA \\ ${ }^{b}$ Department of Electrical and Computer Engineering, University of California, Santa Barbara, \\ 93106 USA
}

\begin{abstract}
The performance of $\operatorname{In}_{\mathrm{x}} \mathrm{Ga}_{1-\mathrm{x}} \mathrm{N}$-based $m$-plane LEDs and laser diodes grown by metalorganic chemical vapor deposition on bulk GaN substrates is currently limited by lower indium uptake and inhomogeneous linewidth broadening in the blue spectrum compared to semipolar planes and $c$-plane. Linewidth broadening is partially attributed to inhomogeneous indium composition that is associated with template morphology. We investigate the morphological evolution of homoepitaxial GaN growth on bulk $m$-plane substrates in three co-loaded miscut orientations: nominally on-axis, $1^{\circ}$ in the $-\mathbf{c}$-direction (-c-miscut), and $1^{\circ}$ in the a-direction (a-miscut). Atomic force microscopy reveals four-sided pyramidal hillocks for on-axis growth with faces inclined toward the [11 $\overline{2} 0]$ a-axis ( $a$-faces) and the [0001] c-axis ( $c$-faces). The $a$-faces exhibit steps oriented in an $a+c$ direction with longer terrace widths than the $c$-face steps. The $-\mathbf{c}$ miscut template growth sometimes forms diagonal striations, characterized by regions with stable $a+c$ step direction. The a-miscut template growth exhibits meandering steps oriented in the $a+/-c$ directions that bunch to form diagonal striations. These results reveal that c-direction steps are unstable compared to $a+/-c$ directions. We further demonstrate that $m$-plane GaN substrates with combined $a+c$ miscut lead to narrower $\operatorname{In}_{\mathrm{x}} \mathrm{Ga}_{1-\mathrm{x}} \mathrm{N}$ photoluminescence emission spectra in blue with enhanced indium incorporation.
\end{abstract}

\section{Keywords}

A1. Crystal morphology

A1. Atomic force microscopy

A3. Metal organic chemical vapor deposition

B1. Nitrides

B2. Semiconducting III-V materials

B3. Light emitting diodes

\footnotetext{
${ }^{1}$ Corresponding author, e-mail address: lkuritzky@umail.ucsb.edu
} 


\section{INTRODUCTION}

(In/Al)GaN-based LED and laser diode (LD) structures grown by metalorganic chemical vapor deposition (MOCVD) have already seen commercial success for visible light emission in the violet up to the green spectral regions. The nonpolar $m$-plane crystal orientation of $\mathrm{GaN}$ offers advantages for visible emitters over the more commonly used basal $c$-plane orientation of $\mathrm{GaN}$ due to the absence of polarization-related electric fields in the growth direction. Thus, $m$ plane GaN-based heterostructures avoid the quantum-confined Stark effect that reduces radiative recombination rates, blue-shifts the emission spectrum with increasing injection current, and necessitates structures with very narrow well widths $(<3 \mathrm{~nm})$ as in $c$-plane LEDs and LDs. The device performance of $m$-plane light emitters is also favored by low threading dislocation (TD) densities $\left(\sim 10^{5}\right)$ and reduced deep level defect states ${ }^{1}$ on lattice-matched bulk GaN substrates. Due to the in-plane asymmetry of the $m$-plane crystal orientation, the valence band (VB) maxima are non-degenerate at the $\Gamma$ point for coherently strained $\operatorname{In}_{\mathrm{x}} \mathrm{Ga}_{1-\mathrm{x}} \mathrm{N}$ layers and the VB separation increases with increasing In composition, $x$. The VBs near $\Gamma$ take on the nitrogen p-orbital nature and thus strained $m$-plane quantum wells (QWs) can emit highly polarized light. ${ }^{2,3}$

High performance $m$-plane LEDs and LDs have been reported in literature, ${ }^{4}$ but broad spectral emission for blue-emitting InGaN QWs, poor indium uptake, and stacking fault generation in green LEDs and LD structures limit the progress for longer wavelength $m$-plane devices. Previous demonstrations of $m$-plane LEDs and LDs in the blue spectrum have shown poor spectral emission characteristics, including broad photoluminescence (PL) emission that is sometimes composed of multiple peaks. ${ }^{5,6}$ Some luminescence broadening is expected due to increasing alloy composition in the blue-emitting InGaN QWs, but excessive broadening or multiple peaks are possibly due to indium fluctuations, QW inhomogeneities, ${ }^{3}$ inherent morphological instabilities or unoptimized growth conditions. Thermalization of higher energy valence band states may also contribute to broadened emission. ${ }^{7}$ Narrow spectral emission often signifies high compositional uniformity of InGaN layers and high crystal quality. For a laser diode, narrow spontaneous spectral emission should lead to higher gain in the lasing mode and reduced lasing threshold. ${ }^{8}$ Nonpolar $m$-plane already has the highest calculated gain of any GaN crystal orientation, ${ }^{3}$ so identifying the origin of the excessive luminescence broadening and resolving this issue for blue and longer wavelengths should greatly enhance nonpolar device performance.

Co-loaded MOCVD growth studies have shown that $m$-plane GaN also suffers from reduced indium incorporation compared to QW structures on $c$-plane and semipolar planes. ${ }^{9}$ Thus, InGaN QW and quantum barrier growth on $m$-plane GaN require lower growth temperatures compared to $c$-plane and semipolar planes to incorporate sufficient indium for QW emission in the blue and green. Lower growth temperatures often lead to increased impurity uptake and formation of defect states in MOCVD growth, including nonradiative centers in the quantum barrier layers. ${ }^{10}$ Basal plane stacking fault formation for high indium composition green-emitting InGaN QW layers on $m$-plane substrates has been attributed to growth errors at low growth temperatures ${ }^{11}$ and has prevented the demonstration of $m$-plane laser diodes with emission wavelengths longer than $500 \mathrm{~nm} .{ }^{12}$ Relaxation of highly strained InGaN layers by prismatic slip on $m$-planes may also prevent the realization of high quality, high composition InGaN QWs. ${ }^{13}$ Enhancing the indium uptake of $m$-plane InGaN would allow for higher growth temperatures and reduced point defect concentrations in the active region, which could improve the performance of longer wavelength devices. 
Semipolar GaN planes, most notably, $(20 \overline{2} \overline{1})$, already demonstrate high performance blue emission. Yet, a major disadvantage of (20 $\overline{2} \overline{1})$ and other semipolar orientations of $\mathrm{GaN}$ is substrate cost and yield. Most semipolar planes are not naturally occurring during bulk crystal growth, ${ }^{14}$ and $(20 \overline{2} \overline{1})$ bulk substrates must be formed by growing along the c-direction in a thick boule and then slicing and polishing, which results in small substrates and low boule yield. ${ }^{15}$ Nonpolar $m$-plane, however, is a naturally occurring facet in bulk growth, enabling a viable future pathway to boules grown in the $m$-direction and, thus, to large area $m$-plane substrates.

Early research on bulk $m$-plane substrates found that the surface morphology is highly sensitive to growth conditions and substrate orientation. MOCVD growth on nominally on-axis bulk $m$-plane GaN leads to surface morphologies dominated by four-sided pyramidal hillocks. The hillocks result from spiral growth around pinned steps at pre-existing TDs ${ }^{16}$ and form two faces inclined toward the [11 20$]$ a-axis ( $a$-faces) and two faces inclined toward the [0001] c-axis ( $+c$-faces and $-c$-faces). Figure 1 shows an atomic force microscopy (AFM) image of a typical pyramidal hillock formed during $100 \mathrm{~nm}$ of homoepitaxial, unintentionally doped, GaN growth on a nominally on-axis, low defect density, bulk $m$-plane substrate. A single pyramid is outlined to guide the eye and delineate the different facets. Note that straight steps are visible on the $a$ faces of the pyramid in Fig. 1 (b) but not the $+/-c$-faces of the pyramid due to resolution limits of the measurement as well as the AFM scan direction.

Intentional miscuts in either the a-direction (a-miscut) or the -c-direction (-c-miscut) can yield smooth films by suppressing the spiral revolutions that lead to pyramid formation. ${ }^{17}$ In past work, the -c-miscut appeared more stable and subsequently showed improved device performance over nominally on-axis substrates and was thus chosen as the preferred miscut orientation. $^{18}$ 

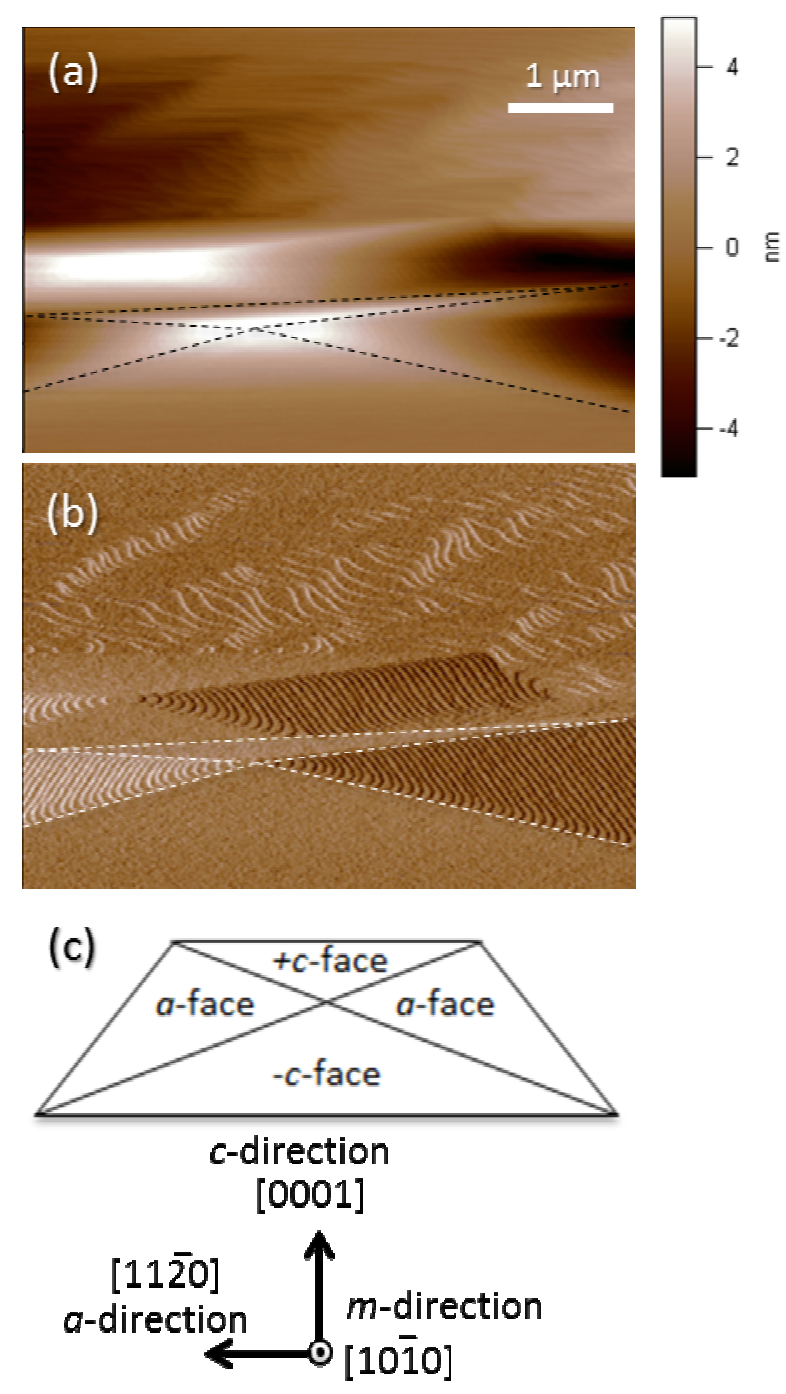

Figure 1. AFM (a) height and (b) amplitude image of pyramidal GaN growth on a nominally on-axis bulk $m$-plane substrate. The nomenclature of the four faces of the pyramid is illustrated in (c).

Recent growth studies of blue-emitting single InGaN QWs grown on on-axis $m$-plane templates with pyramidal hillocks show that the $a$-faces of the pyramids have higher indium uptake, with peak emission wavelengths $40 \mathrm{~nm}$ redshifted compared to the $c$-faces. ${ }^{5}$ The large difference in indium incorporation on spatially separated morphological features leads to dual wavelength PL emission. Further, small miscuts of the $m$-plane substrate $0.5^{\circ}$ in the a-direction or $-1^{\circ}$ in the c-direction showed emission wavelengths similar to the $a$-faces and $c$-faces, respectively, of pyramidal hillocks on the on-axis samples. Specifically, InGaN QWs grown on a-miscut substrates exhibited longer peak wavelengths than QWs grown on -c-miscut substrates. In that study, although both miscuts fully suppressed the pyramidal hillock features, they still exhibited diagonal striations that were visible in AFM. These striations were associated with non-uniformities in indium incorporation, visible in monochromatic cathodoluminescence imaging, which also resulted in broadened PL emission for both miscut orientations. ${ }^{5}$ 
In this paper, we provide details on the morphological evolution and step structure of $m$ plane homoepitaxial films grown on co-loaded bulk substrates with orientations nominally onaxis, $1^{\circ}$-c-miscut, and $1^{\circ}$ a-miscut. We subsequently demonstrate QW structures grown on intentionally miscut $m$-plane substrates with components in both the a-direction and in the cdirection ("double miscuts"). These QWs exhibit narrower photoluminescence linewidth and increased indium incorporation compared to growth on on-axis or -c-miscut substrates.

\section{EXPERIMENTAL}

All growths in this study were performed by atmospheric pressure MOCVD on bulk $m$ plane GaN substrates from Mitsubishi Chemical Corporation. Three separate orientations were used: nominally on-axis; $1^{\circ}$-c-miscut; and $1^{\circ}$ a-miscut. Actual miscuts in the $(\mathbf{c}, \mathbf{a})$ directions are reported as $\left(-0.21^{\circ}, 0.04^{\circ}\right),\left(-0.87^{\circ}, 0.01^{\circ}\right)$ and $\left(-0.26^{\circ}, 1.01^{\circ}\right)$, respectively. In separate studies, we have verified the vendor specific miscut with high resolution X-ray diffraction (typically within $0.01^{\circ}$ of the specified value). ${ }^{19}$

Unintentionally doped (UID) GaN template layers were grown with standard growth conditions in $\mathrm{N}_{2}$ carrier gas, ${ }^{20}$ employing $\mathrm{TMGa}$ and $\mathrm{NH}_{3}$ as group III and $\mathrm{V}$ precursors, respectively. Substrates with orientations nominally on-axis, $1^{\circ}-\mathbf{c}$-miscut, and $1^{\circ} \mathbf{a}$-miscut were co-loaded for all template growths, which were performed at a susceptor temperature of $1100{ }^{\circ} \mathrm{C}$. The templates were grown for four different times to study the morphological evolution: 30 seconds, 2 minutes, 10 minutes and 39 minutes. These times correspond to layer thicknesses of approximately: $25 \mathrm{~nm} ; 100 \mathrm{~nm} ; 500 \mathrm{~nm}$; and $2 \mu \mathrm{m}$, respectively, based on growth rates estimated from separately grown samples measured by X-ray diffraction. The surface morphology for each sample was analyzed with an Asylum MFP-3D AFM in tapping mode with scan sizes ranging from $0.64 \mu \mathrm{m}^{2}$ to $900 \mu \mathrm{m}^{2}$. We include height images, which map the changes in AFM tip height across the sample, a measure of surface morphology. We also include amplitude images, which map the magnitude of deflection in the AFM tip as it interacts with the surface, as this more clearly shows the vicinal step features. ${ }^{21}$

Single QW (SQW) growth structures consisted of $2 \mu \mathrm{m}$ of UID GaN template grown in the same conditions as above, plus $20 \mathrm{~nm}$ of UID GaN barrier, $10 \mathrm{~nm}$ of UID InGaN QW and 10 nm UID GaN cap layer which were grown with TEGa as the group III precursor and TMIn for the QW layer. The QW layer was grown at $810^{\circ} \mathrm{C}$. Several "double miscut" substrate orientations, for which the miscut has both a c-direction component and an a-direction component, were co-loaded: $\left(+0.210^{\circ} / / \mathbf{c}, 0.51^{\circ} / / \mathbf{a}\right),\left(+0.71^{\circ} / / \mathbf{c}, 0.99^{\circ} / / \mathbf{a}\right),\left(+1.41^{\circ} / / \mathbf{c}, 2.00^{\circ}\right.$ $/ / \mathbf{a})$, and $\left(-1^{\circ} / / \mathbf{c}\right)$. PL spectra were measured by topside illumination of the samples at room temperature with a $325 \mathrm{~nm} \mathrm{HeCd}$ excitation source with an intensity of $4 \mathrm{~W} / \mathrm{cm}^{2}$. The peak wavelengths are reported, and the linewidths are given in terms of full-width-at-half-maximum (FWHM) determined by Gaussian fitting.

\section{RESULTS}

\subsection{Template evolution of on-axis $m$-plane}

The morphological evolution of UID GaN template layers grown on nominally on-axis $m$-plane substrates are shown in Fig. 2. These images show the evolution of characteristic spiral hillock features for increasing GaN thickness. 

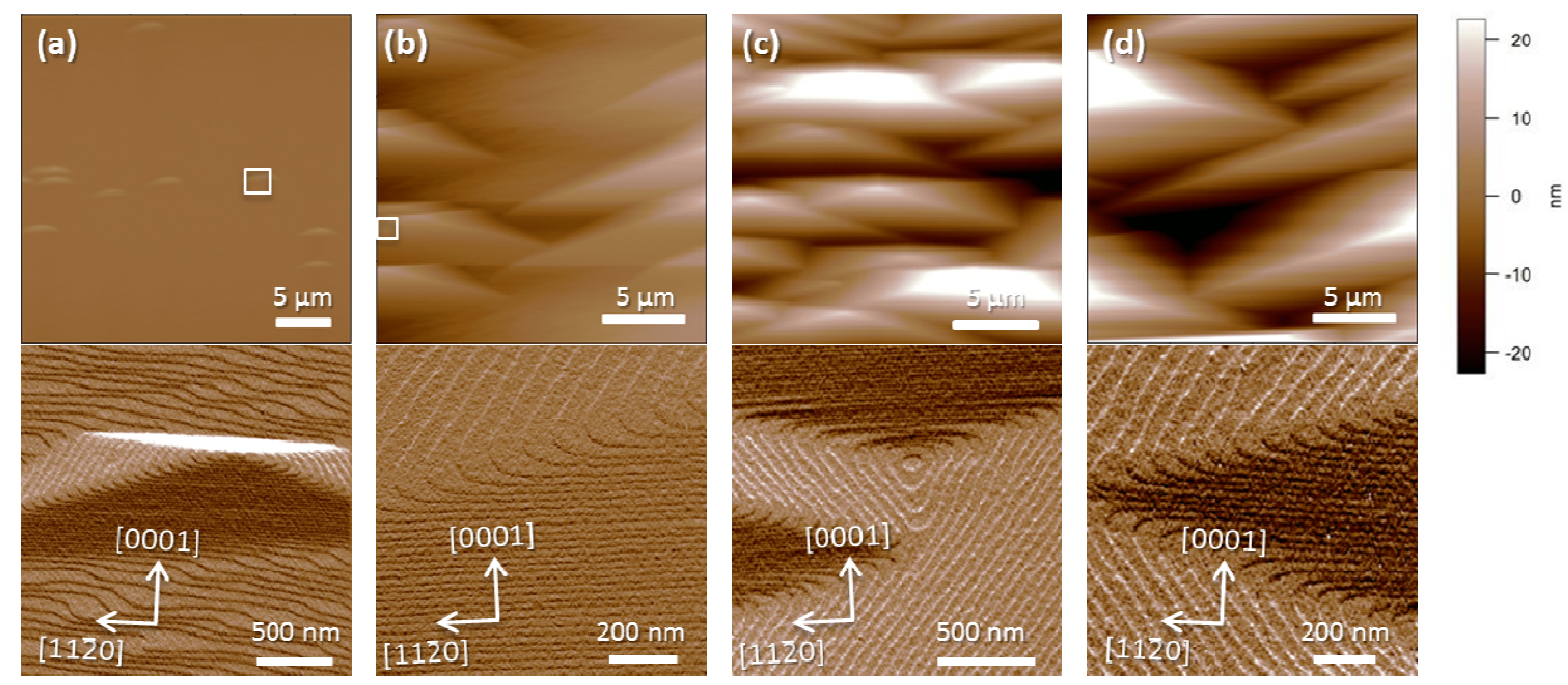

Figure 2. AFM images for homoepitaxial growth on nominally on-axis $\boldsymbol{m}$-plane substrates. The upper panels show large area $\left(400-900 \mu^{2}\right)$ height images for film thicknesses of approximately (a) $25 \mathrm{~nm}$; (b) $100 \mathrm{~nm}$; (c) $500 \mathrm{~nm}$; and (d) $2 \mu \mathrm{m}$. The lower panels show small area (1-4 $\mu^{2}$ ) amplitude images of (a) early hillock formation for $25 \mathrm{~nm}$ film; (b) detail of the $a$ - and $-c$-face edge of a pyramid for $100 \mathrm{~nm}$ thick film; (c) defect free depression between hillocks for $500 \mathrm{~nm}$ thick film; (d) intersection of $a$ - and $-c$-faces.

Figure 2a captures the beginning of hillock formation after 30 seconds of high temperature GaN template growth. The hillock density in this AFM image is $\sim 6 \times 10^{5} \mathrm{~cm}^{-2}$, which is the same order of magnitude as the reported TD density of the bulk $m$-plane substrates. $^{22}$ The dimensions of the hillocks at this stage are approximately $5 \mathrm{~nm}$ tall, $3 \mu \mathrm{m}$ wide in the a-direction and $1 \mu \mathrm{m}$ wide in the c-direction. The RMS roughness of the hillock-free areas of the surface is $0.5 \mathrm{~nm}$ and is composed of meandering steps with line directions approximately along the a-axis, where the line direction is defined as parallel to the step edge. The hillock-free surface exhibits localized regions of steps with mixed a- and $+/-\mathbf{c}$-components of the line direction (henceforth described as $a+c$ or $a-c$ step directions), where the a- and c-components are not necessarily equal in magnitude, as shown in the lower panel of Fig. 2a.

The steps on the $-c$-face of the 4-sided pyramids are oriented in the a-direction, whereas the steps on the $a$-faces have an $a+c$ step direction, oriented approximately $40^{\circ}$ from the c-axis. The angle of the $a$-face steps corresponds to a ratio of approximately 1.25 for the a-component to the c-component of the line direction. Steps on the $a$-faces are straight with evenly spaced terrace widths, indicating stable step flow in this $a+c$ direction.

For thicker template layers, the hillock features grow larger in size, yet the shape of the pyramidal hillock remains unchanged. After 2 minutes $(\sim 100 \mathrm{~nm})$ of growth, the hillocks expand to nearly $20 \mu \mathrm{m}$ in length in the $a$-direction, as shown in Fig. 2b. AFM line scans (not shown), give average terrace widths of the $a$-face and $-c$-face steps of approximately $80 \mathrm{~nm}$ and $25 \mathrm{~nm}$, respectively, and the approximate slope angles are $0.2^{\circ}$ and $0.5^{\circ}$, respectively. The step orientations are shown in more detail in the lower half of Fig. $2 b$, which highlights the $a$-face (top) and $-c$-face (bottom) of a hillock feature.

In thicker GaN layers, the hillock features expand across the entire growth surface, as in Figs. $2 \mathrm{c}$ and $2 \mathrm{~d}$. The sample surface exhibits laterally separated regions of $a$-faces with wide 
terrace widths and shallow slope angles, and $+/-c$-faces with short terrace widths and steeper slope angles. The intersections of $a$-face and $c$-face steps between hillocks form defect-free triangular depressions, shown in the small area (1-4 $\left.\mu^{2}{ }^{2}\right)$ scans of Figs. $2 \mathrm{c}$ and $2 \mathrm{~d}$.

These results indicate that the relative step orientations and terrace widths on the hillock faces remain stable throughout film growth. The preferred vicinal step orientation on the $-c$-face appears to be in the a-direction as expected, but the $a$-faces appear to prefer step direction with a and $\mathbf{c}$ components, rather than a pure c-direction steps.

\subsection{Template evolution of -c-miscut $m$-plane}

Previous studies have demonstrated that vicinal step flow resulting from a $1^{\circ}$-c-miscut suppresses formation of the pyramidal hillocks that are characteristic of on-axis $m$-plane homoepitaxial growth. ${ }^{16,17}$ Despite the absence of pyramids, the emission spectrum from InGaN QWs grown on this miscut can still exhibit broad linewidth, sometimes double-peaked, in the blue spectrum. The emission characteristics have been at least partially attributed to variable indium incorporation at shallow diagonal striations that sometimes emerge in template growth. ${ }^{5}$

Here, we describe the morphological evolution of these diagonal striations in homoepitaxial UID GaN template layers grown on $1^{\circ}$-c-miscut $m$-plane substrates (Fig. 3).
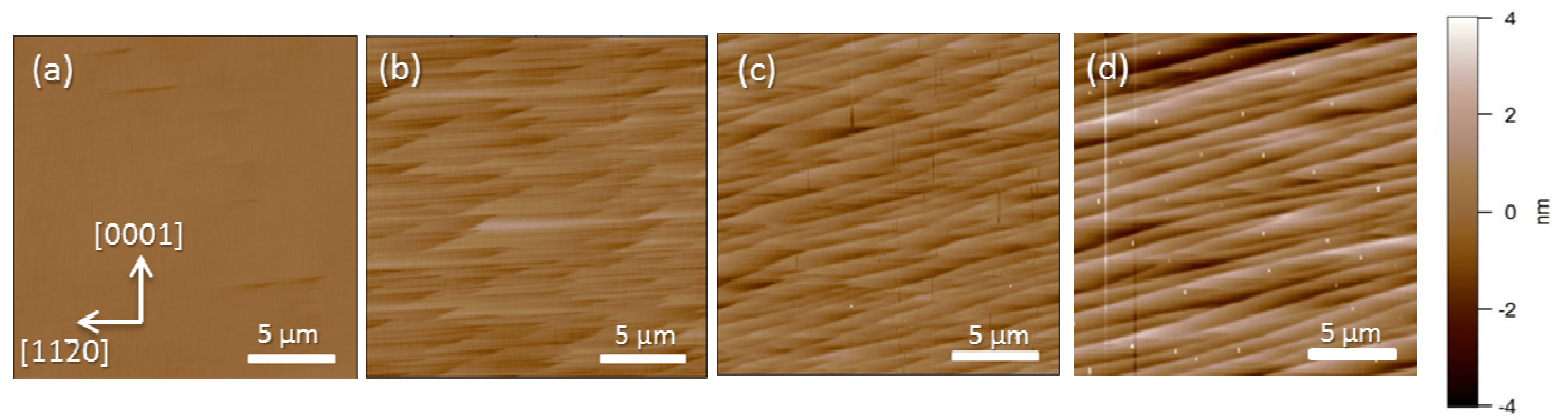

Figure 3. Large area $\left(400 \mathrm{\mu m}^{2}\right)$ AFM height images of GaN grown on $1^{\circ}-\mathrm{c}$-miscut substrates with nominal film thicknesses of (a) $25 \mathrm{~nm}$, (b) $100 \mathrm{~nm}$, (c) $500 \mathrm{~nm}$, and (d) $2 \mu \mathrm{m}$. Shallow grooves in the thin film (a) propagate into diagonal striations in the thicker films (b)-(d).

Thin template growths (Fig. 3a) exhibit shallow disperse grooves. With further growth, diagonal striations appear on the surface, as shown in Figs. 3b-3d. The RMS roughness of the 2 $\mu \mathrm{m}$ template Fig. 3d was $1.23 \mathrm{~nm}$ for a $900 \mu \mathrm{m}^{2}$ scan.

Smaller area AFM images $\left(\sim 40 \mu^{2}\right)$ reveal that the diagonal striations are composed of thin kinked regions of the step flow with monolayer high steps with an $a+c$ line direction. On the remainder of the surface, however, the steps have an a-direction (Fig. 4). Extrinsic perturbations at the substrate surface such as defects, impurities or roughness may be responsible for the nucleation of striations. Intrinsic perturbations such as the Ehrlich-Schwoebel (ES) effect, may also cause growth instabilities due to asymmetric growth kinetics at step features. The relative strength of an ES barrier may depend on growth conditions such as temperature, and may depend on crystallographic orientation. ${ }^{23}$ The effects of ES barriers in this material system are a topic of further study. 


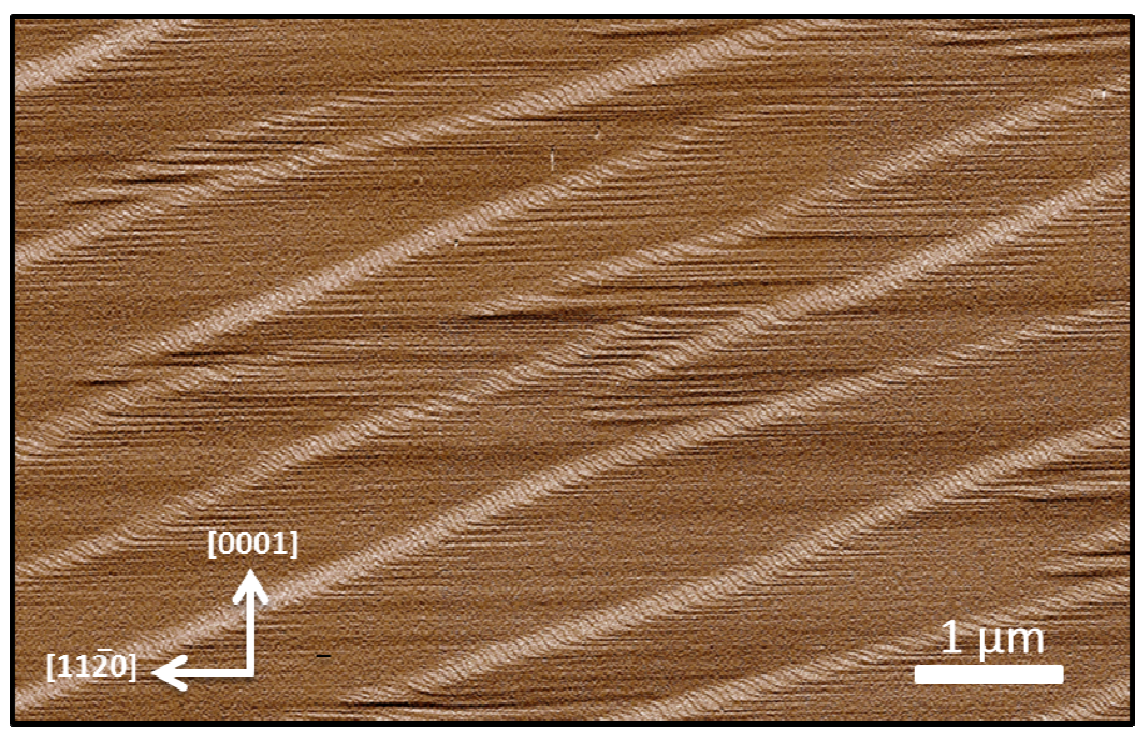

Figure 4. Amplitude AFM image of the surface morphology showing long striations after approximately $100 \mathrm{~nm}$ of growth on $1^{\circ}$-c-miscut $m$-plane substrates.

Typically, a step flow region with high curvature, such as one of these striated regions, will straighten out as growth proceeds due to the line tension in the step. However, these striations appear to propagate over the substrate surface without straightening for thicker films, forming elongated regions where the steps align in an $a+c$ orientation as shown in Fig. 4. The striations may be stabilized by a stable $a+c$ step orientation.

Stable progression of striations is also shown for templates grown on similar substrate miscuts under different growth conditions, which reveal the presence of 'frustrated spirals'. A frustrated spiral is formed by the initiation of a spiral ramp at a TD. The presence of vicinal step flow prevents the spiral from making a full revolution, which suppresses the formation of complete spirals. The frustrated spiral, however, may be the origin of mixed orientation steps as shown in Fig. 5 (note that the sample in Fig. 5a was grown in $3 \mathrm{sccm}$ of TMGa for 4.7 minutes at $1000{ }^{\circ} \mathrm{C}$ and the sample in Fig. 5b, was grown in $7 \mathrm{sccm}$ of TMGa for 2 minutes at $1050{ }^{\circ} \mathrm{C}-$ these conditions differ slightly from other samples reported in this work). 

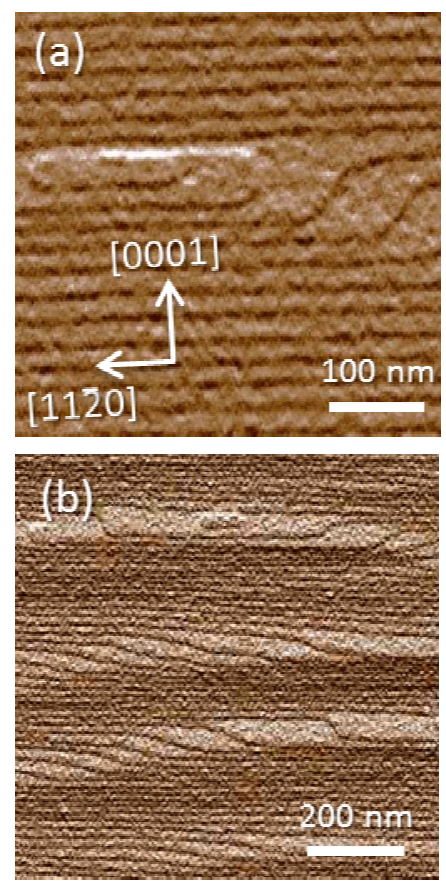

Figure 5. Small area (1-4 $\mu^{2}$ ) amplitude AFM images for thin homoepitaxial template layers grown on $1^{\circ}$-c-miscut $m$-plane bulk substrates, capturing (a) frustrated spiral formation and (b) regions of kinked steps.

The AFM scan in Fig. 5a captures the formation of a frustrated spiral. While parallel steps in the a-direction cover most of the sample, a perturbation caused by the frustrated spiral can disrupt the step direction and cause a kink in the step that can propagate over the substrate surface, as shown in Fig. 5b. The diagonal striations due to a frustrated spiral might be mitigated with increased miscut angle in the -c-direction, which should narrow the terrace spacing between steps along the c-axis and prevent the spiral from initiating. ${ }^{16}$ Striations caused by other surface perturbations may perhaps be mitigated by optimized growth conditions.

\subsection{Template evolution of a-miscut $m$-plane}

Pyramidal hillocks on $m$-plane GaN may also be suppressed by small miscuts toward the adirection, as previous growth studies have found. AFM images of the morphological evolution of GaN template layers grown on nominally $1^{\circ}$ a-miscut substrates are shown in Fig. 6. 

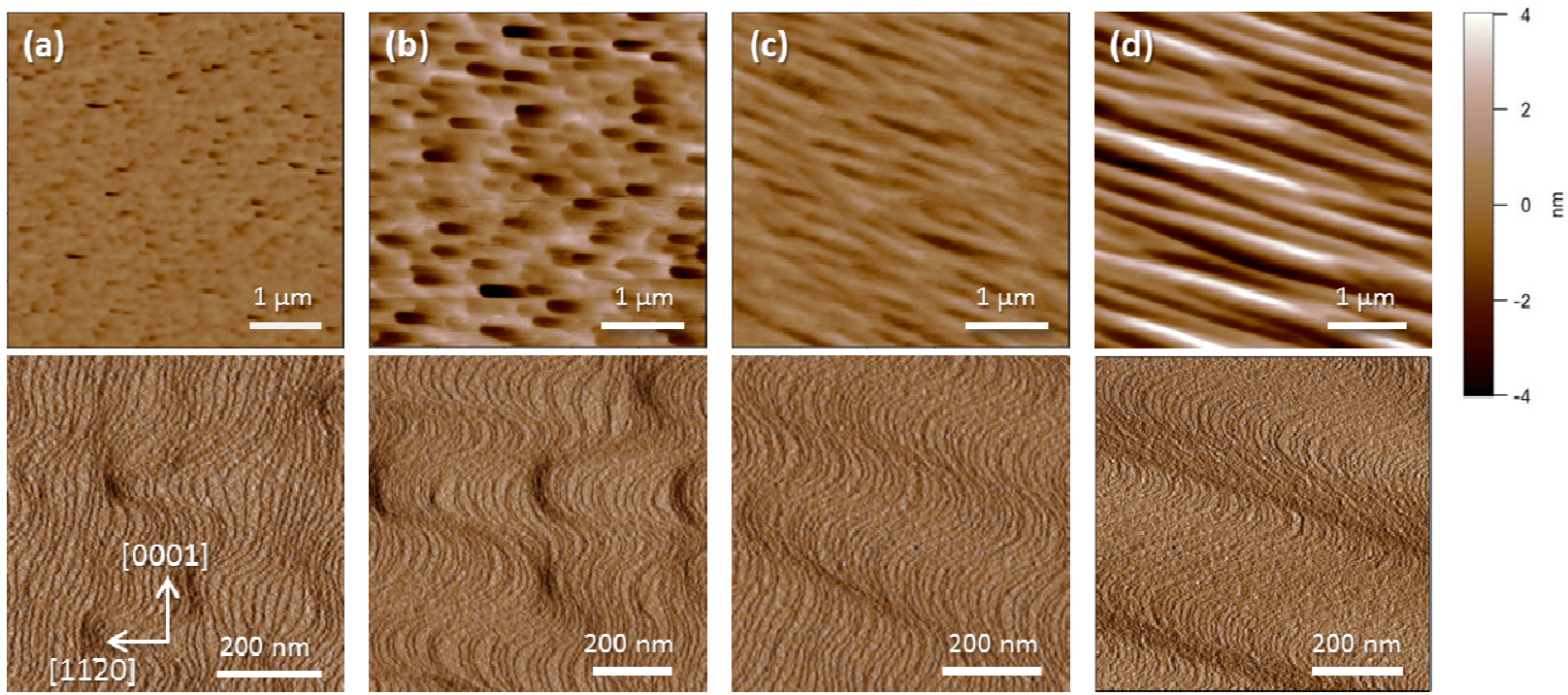

Figure 6. AFM images of the morphological evolution of homoepitaxial growth on $1^{\circ}$ amiscut substrates for approximate film thicknesses: (a) $25 \mathrm{~nm}$, (b) $100 \mathrm{~nm}$, (c) $500 \mathrm{~nm}$, and (d) $2 \mu \mathrm{m}$. The upper panels show $25 \mu^{2}$ height images, and the lower panels show 0.64 $\mu^{2}$ amplitude images, which more clearly reveal the step structure in each sample.

For the shortest growth time of 30s, Fig. 6, the surface is characterized by shallow depressions on the surface. The lower panel of Fig. 6a shows that the vicinal steps for this sample have a meandering profile, with some areas of step-bunching. For thicker GaN layers, the depressions become larger (Fig. 6b) and give way to diagonal striations, as shown in Figs. 6c and $6 \mathrm{~d}$. The amplitude images of these samples reveal that the steps are not parallel to the cdirection, but instead appear to favor the $a+c$ direction or $a-c$ direction. These S-shaped steps align together to form diagonal striations visible in thicker films. The RMS roughness for a 0.64 $\mu \mathrm{m}^{2}$ scan size increases from $0.36 \mathrm{~nm}$ to $1.74 \mathrm{~nm}$ for the $25 \mathrm{~nm}$ thick film and the $2 \mu \mathrm{m}$ thick film, respectively. As was the case for $-\mathbf{c}$-miscuts, a-miscut samples with diagonal striations may lead to broad spectral emission due to differences in indium incorporation along differently oriented steps. ${ }^{5}$

These data demonstrate that $\mathbf{c}$-direction steps are unstable and the meandering steps may be a result of the c-direction steps decomposing into components of $a+c$ and $a-c$ directions, which have longer step length, but apparently lower energy. This observation is consistent with the observations on both on-axis and -c-miscut $m$-plane, which appear to favor the formation of steps in $a+c$ and $a-c$ orientations over formation of pure $\mathbf{c}$-oriented steps. Pure c-direction steps have not yet been observed for any miscut or growth condition in our studies.

Unlike -c-miscut $m$-plane, for which the diagonal striations might be mitigated with increased miscut or improved growth conditions, these results indicate that pure a-miscut $m$ plane will always lead to homoepitaxial surfaces with mixed step orientations and high surface roughness.

\subsection{Preliminary Studies of SQW growth on combined $a+c$ miscuts}

A co-loaded SQW growth was performed with bulk $m$-plane substrates miscut in a combined $a+c$ direction with a constant ratio $\sim 1.25 \mathrm{a} / \mathbf{c}$, as suggested by the step orientations in the AFM data shown above. Three different miscuts were selected for SQW growth: $\left(+0.210^{\circ} / /\right.$ 
$\left.\mathbf{c}, 0.51^{\circ} / / \mathbf{~ a}\right),\left(+0.71^{\circ} / / \mathbf{c}, 0.99^{\circ} / / \mathbf{a}\right),\left(+1.41^{\circ} / / \mathbf{c}, 2.00^{\circ} / / \mathbf{a}\right)$ and were co-loaded with a standard miscut substrate $\left(-1^{\circ} / / \mathbf{c}\right)$. Preliminary PL results of this co-loaded study are shown in Fig. 7. The standard -c-miscut sample (a) shows narrow emission, but with a short peak wavelength in violet at $412 \mathrm{~nm}$ with FWHM of $14.5 \mathrm{~nm}$. Although the linewidth is narrow in violet, this miscut has previously been observed to yield broader linewidths in blue, in excess of $40 \mathrm{~nm}$ FWHM near $450 \mathrm{~nm} .^{5}$ The three $a+c$ miscut samples all show redshifted wavelengths compared to the c-miscut. The $\left(+0.71^{\circ} / / \mathbf{c}, 0.99^{\circ} / /\right.$ a) miscut substrate in Fig. $7 c$ exhibits a nearly $40 \mathrm{~nm}$ wavelength redshift compared to the standard $1^{\circ}-\mathbf{c}$-miscut. This redshift has been observed repeatedly for substrates containing any a-miscut, whether or not $+/-\mathbf{c}$-miscut is also present. The miscut orientation with the smallest $a+c$ miscut angle shows dual wavelength PL emission (Fig. 7b). The samples with the larger magnitude $a+c$ miscuts show sub-30 nm linewidths in the blue spectrum (Fig. 7c and 7d). This is a marked improvement on previous observations of $>40$ $\mathrm{nm}$ on -c-miscut samples in blue.
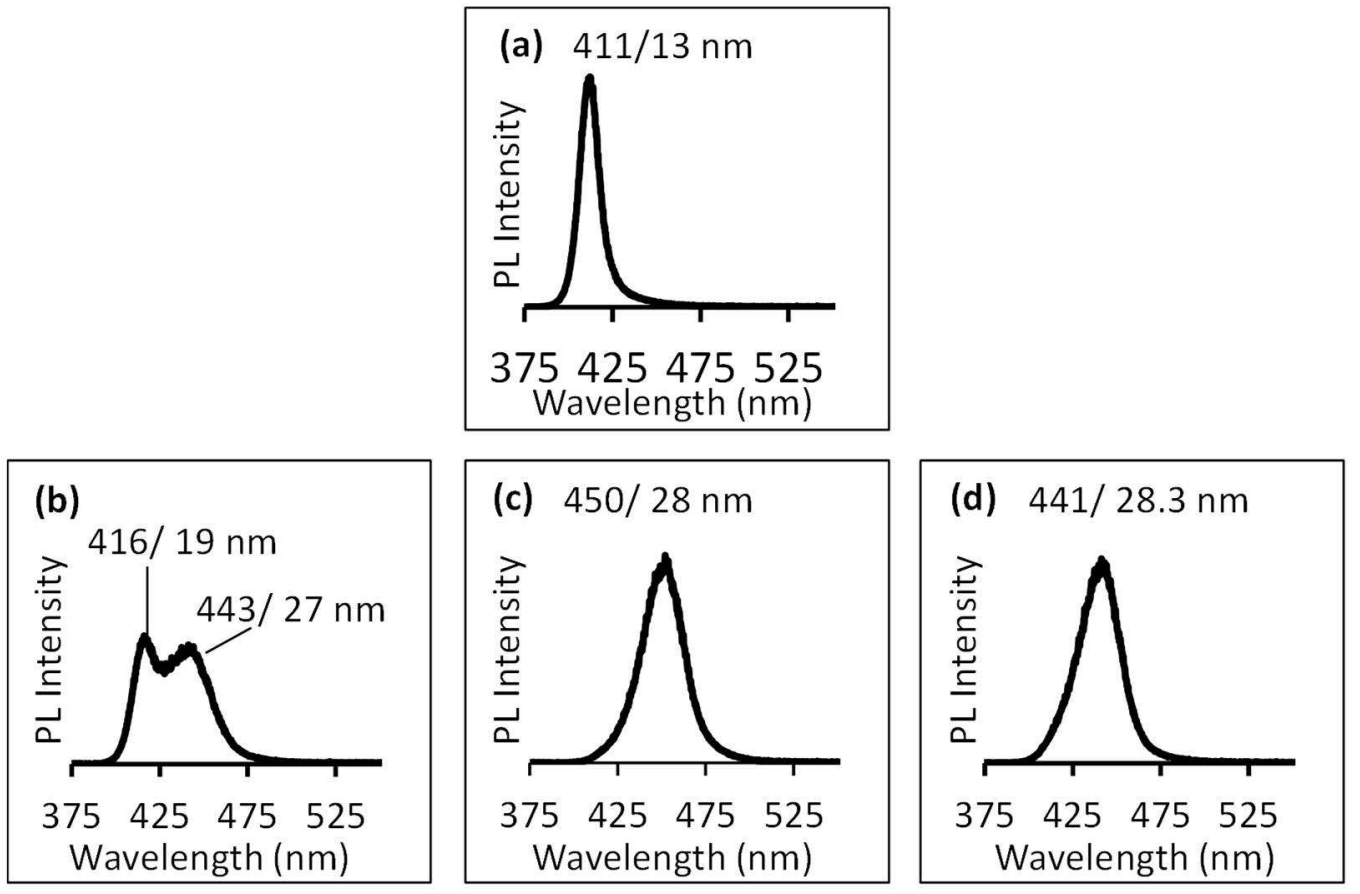

Figure 7. PL spectra for SQW grown on (a) standard $1^{\circ}$-c-miscut; (b) $\left(+0.210^{\circ} / / \mathrm{c}, \mathbf{0 . 5 1}^{\circ} / /\right.$ a); (c) $\left(+0.71^{\circ} / / \mathrm{c}, \mathbf{0 . 9 9 ^ { \circ }} / / \mathrm{a}\right)$; and (d) $\left(+\mathbf{1 . 4 1 ^ { \circ }} / / \mathrm{c}, \mathbf{2 . 0 0 ^ { \circ }} / /\right.$ a). The intensity scale (arbitrary units) is the same for all four plots. The peak and FWHM values are determined by Gaussian fits for each spectrum. The double peak in (b) is fit with two Gaussians.

\section{DISCUSSION}

Previously, we reported ${ }^{5}$ that SQW emission on different faces of the pyramidal hillock features on on-axis $m$-plane may differ by as much as $30 \mathrm{~nm}$. This demonstrates that the pyramid faces incorporate indium differently, causing broad emission in the blue spectral region, composed of dual peaks for single and multiple QW structures. In this paper, we have shown that both the step direction and terrace width are very different on these two faces of pyramidal hillocks. While the $c$-faces steps are aligned along the crystallographic a-direction, the $a$-face 
steps are aligned along an $a+c$ direction and both step orientations appear stable throughout template evolution.

The differences in indium incorporation on the pyramidal hillock faces may be due to both step orientation and terrace width. We propose that step orientation (i.e. miscut direction) plays a larger role in indium incorporation than step spacing (i.e. miscut magnitude) because miscut samples with very different terrace widths yet similar step orientations maintain approximately the same peak emission wavelengths as the on-axis samples. Previously, ${ }^{5}$ it was shown that -c-miscut samples maintained similar peak wavelengths to the $+/-c$-faces of the onaxis pyramids despite shorter terrace widths, and a-miscut showed similar peak wavelength to the $a$-faces. It appears that miscuts that generate vicinal step directions with some component along the c-axis, including both the pure a-miscut and the $a+c$ miscut substrates, give improved indium incorporation.

In this study, $a+c$ and $a-c$ appear to be stable growth directions that emerge naturally in the template growth of all of the co-loaded substrate orientations: on-axis, -c-miscut and amiscut. The $a$-faces of the on-axis pyramidal hillocks exhibit straight, defect-free, steps in the $a+c$ direction. The $+c$ - and $-c$ - faces of the on-axis pyramidal hillocks exhibit steps in the adirection which are also straight and defect-free. The regions surrounding the on-axis pyramids in thin template layer growth show stable meandering steps with $a+c$ and $a-c$ directions. These step orientations also appear on -c-miscut samples where surface perturbations create kinks in the step structure that propagate and lead ultimately to diagonal striations and surface roughness. Finally, diagonal striations for pure a-miscut homoepitaxial growth may be due to the decomposition of c-direction steps into $a+c$ and $a-c$ meandering vicinal step profiles, which also lead to surface striations. Both pyramids and striations affect the indium uniformity of subsequent QW growth and can lead to regions of different indium incorporation and broad, dual wavelength emission. ${ }^{5}$

Preliminary data shows that substrates with miscut in an $a+c$ direction may encourage unimpeded progression of the stable $a+c$ oriented steps that emerge in $m$-plane growth of hillocked on-axis templates, $-\mathbf{c}$-miscut templates and a-miscut templates. These $a+c$ miscut orientations appear to result in more uniform indium incorporation, leading to narrower spectral emission for subsequent SQW growth. Further, the emission is redshifted compared to miscuts in the -c-direction under identical growth conditions, signifying an increase in apparent indium incorporation.

\section{Conclusion}

In this paper, we have shown that broad spectral emission of blue-emitting QW structures on $m$-plane $\mathrm{GaN}$ can be tied to progression of vicinal steps of the underlying template layers. Miscut direction and magnitude as well as template growth conditions determine the overall vicinal step orientation and progression.

Steps oriented in the $a+c$ direction appear naturally in lieu of c-direction steps on all three substrate miscuts: (1) on the $a$-faces of on-axis pyramidal hillock features; (2) propagating in striations generated from surface perturbations in -c-miscuts; and (3) in meandering step flow for a-miscuts, which decomposes from c-direction steps into a combination of $a+c$ and $a-c$ direction steps. These regions with $a+c$ or $a-c$ orientation also incorporate more indium than regions where steps are oriented in the a-direction. We may take advantage of this natural $a+c$ step direction by intentionally miscutting substrates in this orientation. 
Previous work has shown smooth template morphology with miscuts purely in the -cdirection, ${ }^{16,17}$ as well as improved device results ${ }^{18}$ compared to on-axis growths. Competitive LED device results in violet ${ }^{24-26}$ and blue ${ }^{18,27}$ spectra were also demonstrated in this miscut orientation. However, laser thresholds were higher ${ }^{27}$ in the blue spectrum, possibly due to broad spontaneous emission wavelengths. In this paper, preliminary data shows enhanced indium incorporation and narrower spectral width by controlling the vicinal step features with $a+c$ miscut substrates, which should lead to enhanced performance for nonpolar blue LEDs and laser diodes. Enhanced indium incorporation also enables active region growth at higher temperatures, which may improve material quality and device performance for longer wavelength applications.

\section{Acknowledgments}

The authors wish to acknowledge financial support from the Solid State Lighting and Energy Center (SSLEC) at the University of California, Santa Barbara (UCSB), the Center for Energy Efficient Materials, an Energy Frontier Research Center funded by DOE, Office of Science, BES under Award \# DE-SC0001009 A portion of this work was done in the MRL shared experimental facilities, a member of the NSF-funded Materials Research Facilities Network supported by the MRSEC Program of the NSF under Award no. DMR 1121053. This material is based upon work supported by the National Science Foundation Graduate Research Fellowship Program under Grant No. DGE-1144085. Any opinions, findings, and conclusions or recommendations expressed in this material are those of the authors and do not necessarily reflect the views of the National Science Foundation.

\section{References}

${ }^{1}$ T.A. Henry, A. Armstrong, K.M. Kelchner, S. Nakamura, S.P. DenBaars, and J.S. Speck, Appl. Phys. Lett. 100, 082103 (2012).

${ }^{2}$ D.F. Feezell and M.C. Schmidt, 34, (2009).

${ }^{3}$ W. Scheibenzuber, U. Schwarz, R. Veprek, B. Witzigmann, and A. Hangleiter, Phys. Rev. B 80, 115320 (2009).

${ }^{4}$ S. Nakamura and M. Krames, 101, 2211 (2013).

${ }^{5}$ K. Kelchner, L. Kuritzky, K. Fujito, S. Nakamura, S. DenBaars, and J. Speck, J. Cryst. Growth 382, 80 (2013).

${ }^{6}$ S.F. Chichibu, M. Kagaya, P. Corfdir, J.-D. Ganière, B. Deveaud-Plédran, N. Grandjean, S. Kubo, and K. Fujito, Semicond. Sci. Technol. 27, 024008 (2012).

${ }^{7}$ S. Marcinkevičius, K.M. Kelchner, S. Nakamura, S.P. DenBaars, and J.S. Speck, Appl. Phys. Lett. 102, 101102 (2013).

${ }^{8}$ A. Pourhashemi, R.M. Farrell, M.T. Hardy, P.S. Hsu, K.M. Kelchner, J.S. Speck, S.P. DenBaars, and S. Nakamura, Appl. Phys. Lett. 103, 151112 (2013). 
${ }^{9}$ Y. Zhao, Q. Yan, C.-Y. Huang, S.-C. Huang, P. Shan Hsu, S. Tanaka, C.-C. Pan, Y. Kawaguchi, K. Fujito, C.G. Van de Walle, J.S. Speck, S.P. DenBaars, S. Nakamura, and D. Feezell, Appl. Phys. Lett. 100, 201108 (2012).

${ }^{10}$ A.M. Armstrong, K. Kelchner, S. Nakamura, S.P. DenBaars, and J.S. Speck, Appl. Phys. Lett. 103, 232108 (2013).

${ }^{11}$ F. Wu, Y.-D. Lin, A. Chakraborty, H. Ohta, S.P. DenBaars, S. Nakamura, and J.S. Speck, Appl. Phys. Lett. 96, 231912 (2010).

${ }^{12}$ K. Okamoto, J. Kashiwagi, T. Tanaka, and M. Kubota, Appl. Phys. Lett. 94, 71105 (2009).

${ }^{13}$ T. Hanada, T. Shimada, S.-Y. Ji, K. Hobo, Y. Liu, and T. Matsuoka, Phys. Status Solidi 8, 444 (2011).

${ }^{14}$ B.N. Bryant, A. Hirai, E.C. Young, S. Nakamura, and J.S. Speck, J. Cryst. Growth 369, 14 (2013).

${ }^{15}$ K. Fujito, S. Kubo, and I. Fujimura, MRS Bull. 34, (2009).

${ }^{16}$ R.M. Farrell, D.A. Haeger, X. Chen, C.S. Gallinat, R.W. Davis, M. Cornish, K. Fujito, S. Keller, S.P. DenBaars, and S. Nakamura, Appl. Phys. Lett. 96, (2010).

${ }^{17}$ A. Hirai, Z. Jia, M.C. Schmidt, R.M. Farrell, S.P. DenBaars, S. Nakamura, J.S. Speck, and K. Fujito, Appl. Phys. Lett. 91, 191906 (2007).

${ }^{18}$ Y.-D. Lin, M.T. Hardy, P.S. Hsu, K.M. Kelchner, C.-Y. Huang, D.A. Haeger, R.M. Farrell, K. Fujito, A. Chakraborty, H. Ohta, J.S. Speck, S.P. DenBaars, and S. Nakamura, Appl. Phys. Express 2, 082102 (2009).

${ }^{19}$ E.C. Young. Personal Communication (2014).

${ }^{20}$ R.M. Farrell, D.A. Haeger, X. Chen, M. Iza, A. Hirai, K.M. Kelchner, K. Fujito, A. Chakraborty, S. Keller, S.P. DenBaars, J.S. Speck, and S. Nakamura, J. Cryst. Growth 313, 1 (2010).

${ }^{21}$ J. Li. Asylum Web Tutorial (2014).

${ }^{22}$ K. Fujito, K. Kiyomi, T. Mochizuki, H. Oota, H. Namita, S. Nagao, and I. Fujimura, Phys. Status Solidi 205, 1056 (2008).

${ }^{23}$ M.A. Herman, W. Richter, and H. Sitter, Epitaxy (2010), pp. 344-345.

${ }^{24}$ R. Farrell, D.A. Haeger, P.S. Hsu, M. Schmidt, K. Fujito, D. Feezell, S.P. DenBaars, J. Speck, and S. Nakamura, Appl. Phys. Lett. 99, 171113 (2011). 
${ }^{25}$ R. Farrell, P. Hsu, D. Haeger, K. Fujito, S. DenBaars, J. Speck, and S. Nakamura, Appl. Phys. 96, 231113 (2010).

${ }^{26}$ C. Holder, J.S. Speck, S.P. DenBaars, S. Nakamura, and D. Feezell, Appl. Phys. Express 5, 92104 (2012).

${ }^{27}$ K.M. Kelchner, R.M. Farrell, Y.-D. Lin, P.S. Hsu, M.T. Hardy, F. Wu, D.A. Cohen, H. Ohta, J.S. Speck, S.P. DenBaars, and S. Nakamura, Appl. Phys. Express 3, 092103 (2010). 